\title{
Experimental entanglement distillation of mesoscopic quantum states
}

\author{
RUIFANG DONG ${ }^{1}$, MIKAEL LASSEN ${ }^{1,2}$, JOEL HEERSINK ${ }^{1}$, CHRISTOPH MARQUARDT ${ }^{1}$, RADIM FILIP ${ }^{1,3}$, \\ GERD LEUCHS ${ }^{1}$ AND ULRIK L. ANDERSEN ${ }^{1,2 *}$
}

\author{
${ }^{1}$ Institut für Optik, Information und Photonik, Max-Planck Forschungsgruppe, Universität Erlangen-Nürnberg, Günther-Scharowsky-Str. 1, 91058, Erlangen, Germany \\ ${ }^{2}$ Department of Physics, Technical University of Denmark, Building 309, 2800 Kgs. Lyngby, Denmark \\ ${ }^{3}$ Department of Optics, Palacký University, 17. listopadu 50, 77200 Olomouc, Czech Republic \\ *e-mail: ulrik.andersen@fysik.dtu.dk
}

The distribution of entangled states between distant parties in an optical network is crucial for the successful implementation of various quantum communication protocols such as quantum cryptography, teleportation and dense $\operatorname{coding}^{1-3}$. However, owing to the unavoidable loss in any real optical channel, the distribution of loss-intolerant entangled states is inevitably afflicted by decoherence, which causes a degradation of the transmitted entanglement. To combat the decoherence, entanglement distillation, a process of extracting a small set of highly entangled states from a large set of less entangled states, can be used ${ }^{4-14}$. Here we report on the distillation of deterministically prepared light pulses entangled in continuous variables that have undergone non-Gaussian noise. The entangled light pulses ${ }^{15-17}$ are sent through a lossy channel, where the transmission is varying in time similarly to light propagation in the atmosphere. By using linear optical components and global classical communication, the entanglement is probabilistically increased.

Entanglement distillation has been experimentally demonstrated for spin-1/2 (or qubit) systems exploiting a posteriori generated polarization-entangled states ${ }^{6}$. However, the implementation of a scheme that is capable of distilling entanglement of continuous-variable systems, where information is encoded into mesoscopic carriers such as the quadratures of light modes, has remained an experimental challenge. It has been shown theoretically that if the wavefunction for the canonically conjugate variables of the light mode is Gaussian, entanglement distillation can be done only by using highly nonlinear (thus difficult) operations, no matter whether it is a pure or a mixed state $e^{7-9}$. Several protocols have been put forward ${ }^{10-13}$, and a proof-of-principle experiment on the concentration of entanglement using non-local and non-Gaussian operations has recently been implemented ${ }^{14}$.

In many practical scenarios, however, the transmitted quantum state will be non-Gaussian: one example is the transmission of light through a turbulent atmospheric channel, where the attenuation coefficient will fluctuate in time, thus resulting in a non-Gaussian quantum state ${ }^{18,19}$. Fortunately, as we will show in this letter, it is possible to distil entanglement that has undergone such noise by means of linear optical components, a simple measurementinduced Gaussian operation and classical communication.

We consider an optical field mode succinctly described by its canonically conjugated quadrature amplitudes, which correspond to the real and imaginary parts of the complex field. We denote by $\hat{X}$ the amplitude quadrature and $\hat{P}$ the phase quadrature. The optical field can be represented by a quasiprobability distribution known as the Wigner function $W(X, P)$, where $X$ and $P$ are eigenvalues of $\hat{X}$ and $\hat{P}$. Having two optical fields, described by the quadratures $\left(\hat{X}_{\mathrm{A}}, \hat{P}_{\mathrm{A}}\right)$ and $\left(\hat{X}_{\mathrm{B}}, \hat{P}_{\mathrm{B}}\right)$, the joint state can be described by the joint Wigner function $W\left(X_{\mathrm{A}}, P_{\mathrm{A}}, X_{\mathrm{B}}, P_{\mathrm{B}}\right)$. If this function is Gaussian the joint optical state can be fully characterized by its covariance matrix. For this case the logarithmic negativity (which is an entanglement monotone), denoted by LN, of the state is simply given by

$$
\mathrm{LN}=-\log _{2} \mu_{\min },
$$

where $\mu_{\min }$ is the smallest symplectic eigenvalue of the partial transposed covariance matrix ${ }^{20}$.

Suppose now that one mode of the Gaussian entangled state is sent through a medium with varying attenuation. We consider $N$ different levels of attenuation. After the transmission the state turns into a less entangled or even unentangled state and is described by the convex mixture

$$
W\left(X_{\mathrm{A}}, P_{\mathrm{A}}, X_{\mathrm{B}}, P_{\mathrm{B}}\right)=\sum_{i=1}^{N} p_{i} W_{i}^{\prime}\left(X_{\mathrm{A}}, P_{\mathrm{A}}, X_{\mathrm{B}}, P_{\mathrm{B}}\right),
$$

where $p_{i}$ is the probability of a certain transmittance and the Wigner function $W_{i}^{\prime}$ represents the state in channel $i$ after transmission. The individual constituents of the mixture are all Gaussian functions but the sum is a non-Gaussian function. Because of this non-Gaussianity, distillation can be enabled solely by linear optics and feedforward as illustrated in Fig. 1. The operation consists of a weak measurement (implemented by a $7 \%$ reflecting beam splitter and a homodyne detector measuring $\hat{X}$ ) followed by a probabilistic heralding process, where the remaining state is kept or discarded conditioned on the measurement outcomes. If the outcome of the weak measurement is larger than a specified threshold value, $X_{\text {th }}$ (see Fig. $2 \mathrm{a}$ ), the remaining state is kept $^{21-25}$, thus resulting in probabilistic recovery of the entanglement with a corresponding increase in LN (see Methods section). Note that our protocol cannot distil more entanglement than is contained in the most entangled component of the mixture in equation (2). A notable difference between our distillation approach and the schemes proposed in refs 12,26 is that our procedure relies on single copies of distributed entangled states, whereas the protocols in refs 12,26 are based on at least two copies. 


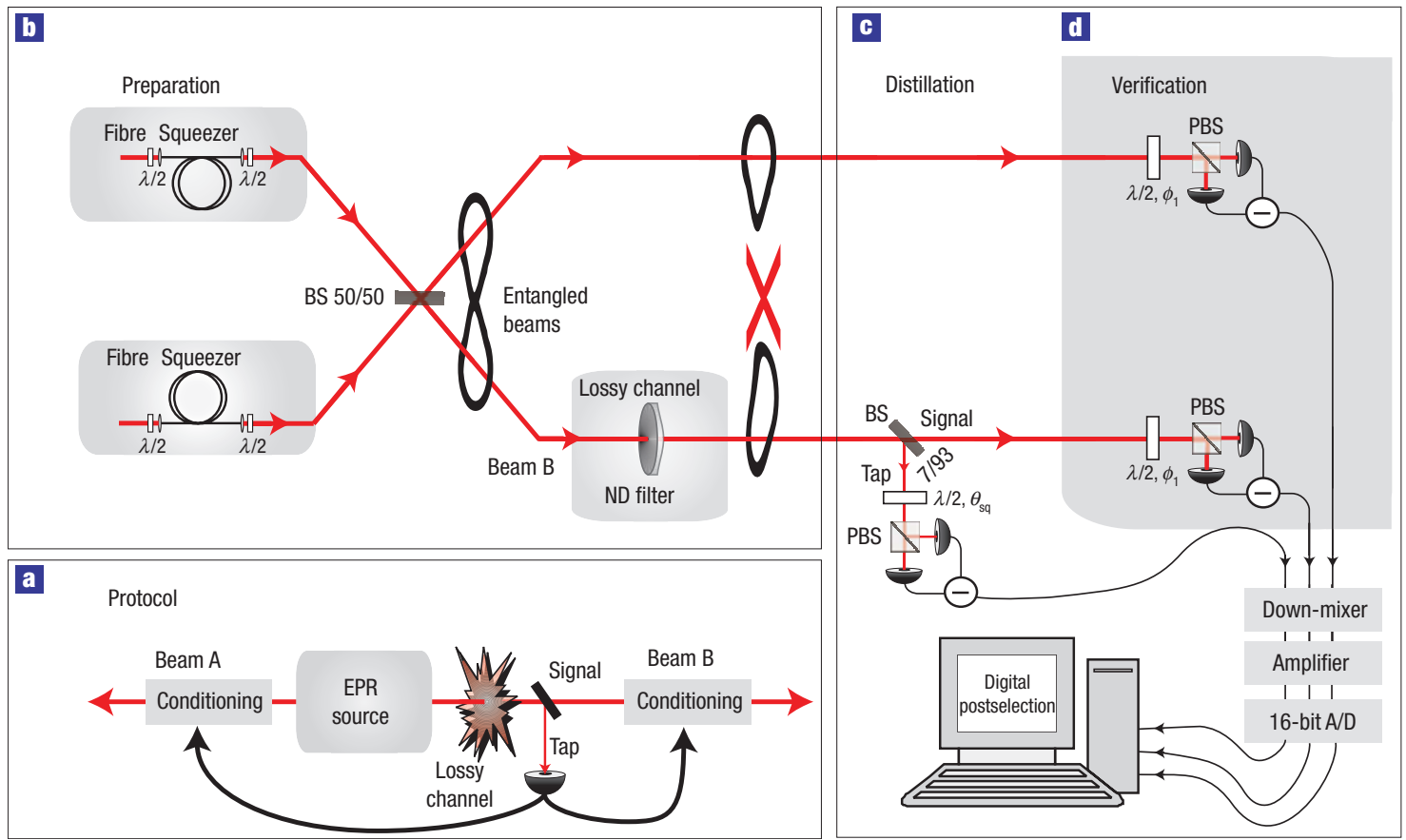

Figure 1 Schematic diagrams of the entanglement distillation protocol and the experimental set-up. a, A weak measurement on beam B diagnoses the state and is subsequently used to herald the highly entangled components of the state. $\mathbf{b}$. The entangled states are prepared by making two polarization-squeezed beams interfere on a $50 / 50$ beam splitter (BS). The lossy channel is implemented by sending beam B through a controllable neutral-density (ND) filter. c, The distillation operation consists of a weak measurement (implemented by a $7 \%$ reflecting beam splitter) and homodyne measurement. $\mathbf{d}$, The verification measurement uses two independent polarization detectors set to measure the conjugate quadratures $\hat{X}$ and $\hat{P}$. The photocurrents are down-mixed, digitized with a fast analog-to-digital (A/D) converter and fed into a computer. (See the Methods section for more details.)
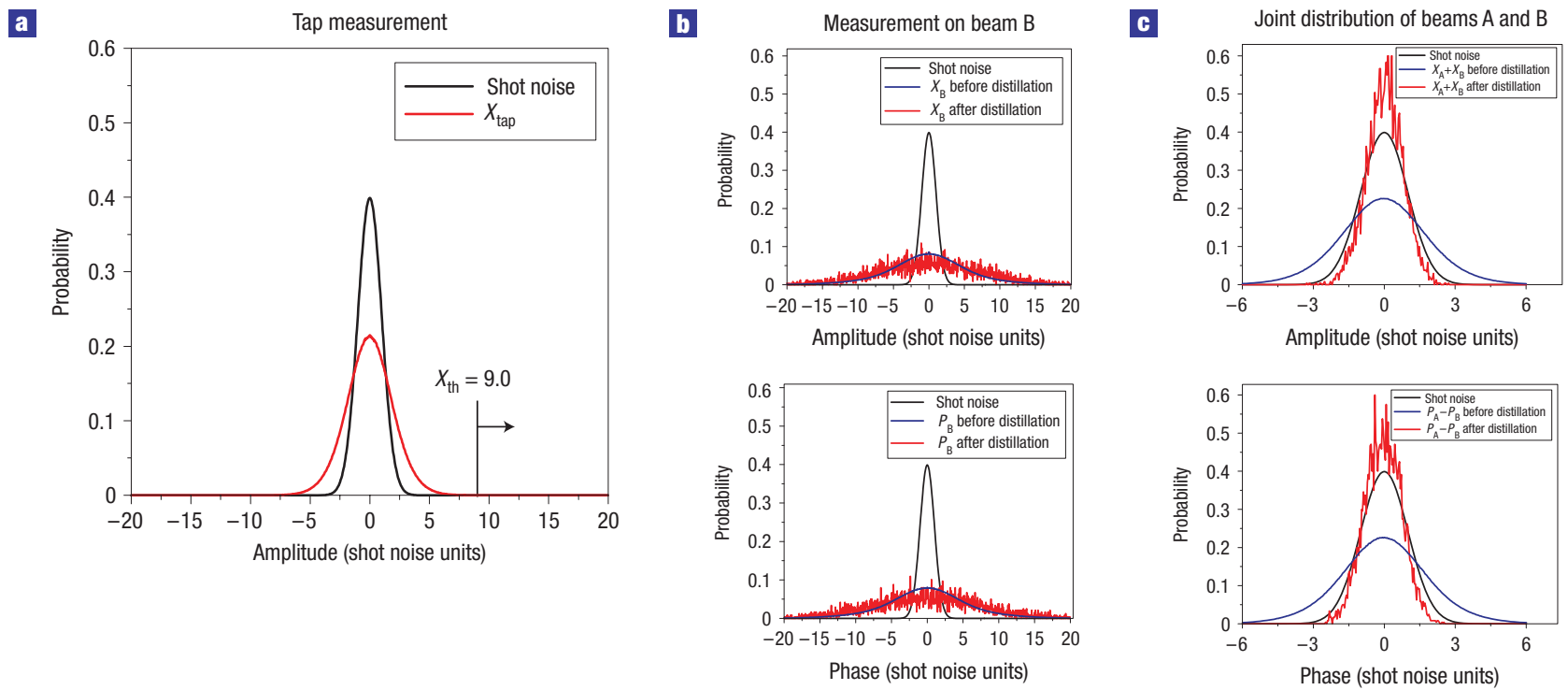

Figure 2 Experimentally measured marginal distributions illustrating the effect of distillation. a, Marginal distribution for the amplitude quadrature in the tap measurement. The vertical line indicates the threshold value chosen for this realization. b,c, Marginal distributions associated with the measurements of $X$ and $P$ of beam $B$ (b) and the joint measurements $X_{\mathrm{A}}+X_{\mathrm{B}}$ and $P_{\mathrm{A}}-P_{\mathrm{B}}(\mathbf{c})$.

The experimental realization is divided into three steps, preparation, distillation and verification, as schematically illustrated in Fig. 1. The entangled states are prepared by interfering two squeezed beams on a 50/50 beam splitter. The squeezed beams are generated by exploiting the Kerr nonlinearity experienced by ultrashort laser pulses in optical fibres ${ }^{16}$. To ease the detection 
a

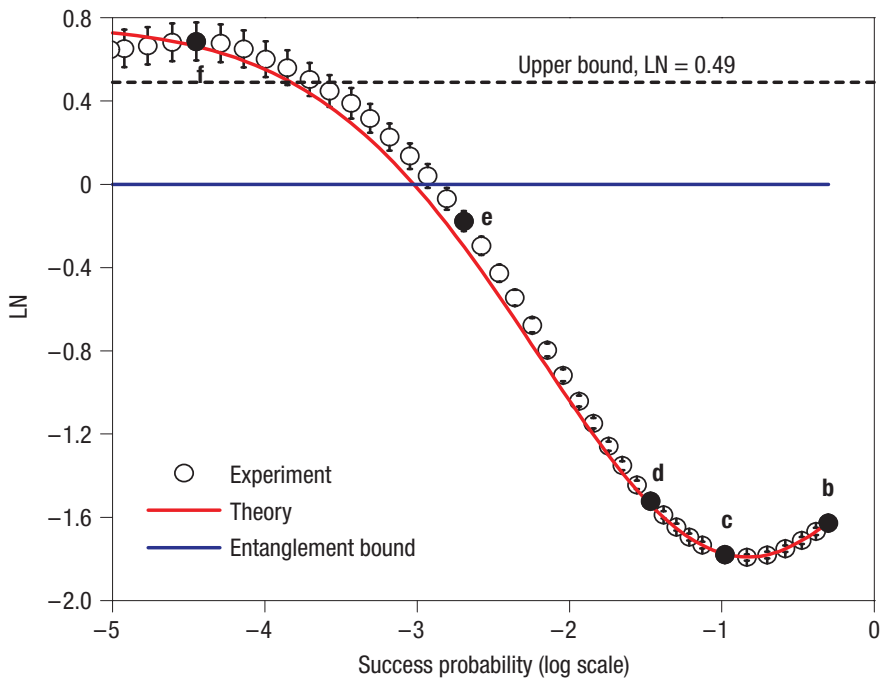

f

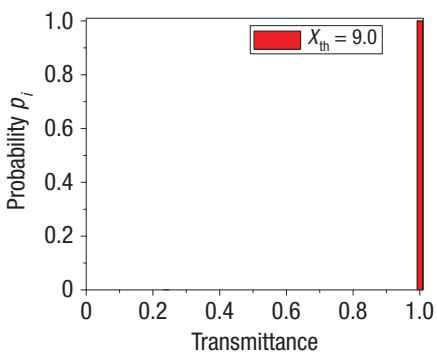

Q

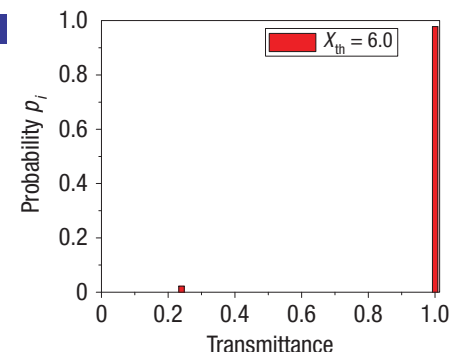

b

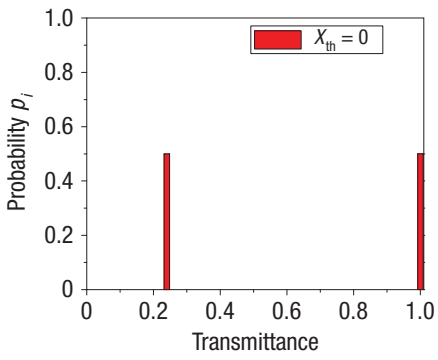

c

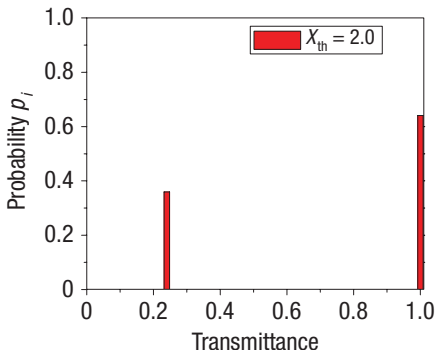

d

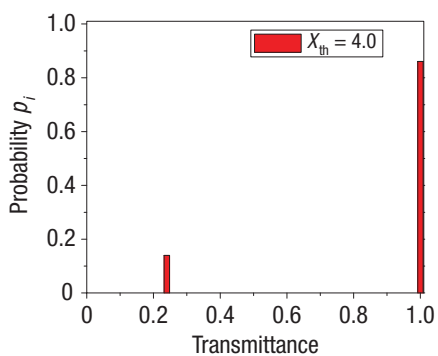

Figure 3 Experimental and theoretical results outlining the distillation of an entangled state from a discrete lossy channel. a, The experimental results are marked by circles and the theoretical prediction is plotted by the red solid line. The bound for Gaussian entanglement is given by the blue line, and the upper bound for total entanglement before distillation is given by the black dashed line. Both bounds are surpassed by the experimental data. The error bars of LN represent the standard deviations, which depend partly on the measurement uncertainties, mainly associated with the finite resolution of the A/D converter, and partly on the statistical uncertainties due to the finite measurement time and the postselection process. $\mathbf{b}-\mathbf{f}$, The weight of the two constituents in the mixed state after distillation for various threshold values is also experimentally investigated. The plots explicitly show the effect of the distillation.

process we produce polarization-squeezed states, which inherently contain bright polarization components that are used as local oscillators in homodyne detection as described in refs 15,17 . Details about the generation and measurement of entanglement can be found in the Methods section. The Gaussian properties of the entangled states are characterized by measuring the entries of the covariance matrix, though setting the intracorrelations (such as $\left.\left\langle\hat{X}_{\mathrm{A}} \hat{P}_{\mathrm{A}}\right\rangle\right)$ to zero by generating near-symmetric states and choosing an appropriate reference frame. From the covariance matrix we compute the smallest symplectic eigenvalue, from which we find the $\mathrm{LN}$ to be $0.76 \pm 0.08$.

We implement the lossy channel by inserting a neutral-density filter with a variable transmittance in one of the entangled beams. The entangled beam is then transmitted through a channel with $N=45$ different levels with corresponding transmittance from 0.1 to 1 in steps of $0.9 / 44$. Combining all these realizations of the experiment, a mixed state such as the one given by equation (2) is formed with the probabilities $p_{i}$ all being identical. However, after the measurement we select the outcomes so as to give the different channels prespecified probability weights. With this technique we can easily implement different transmission scenarios. Distillation of entanglement is demonstrated for two different lossy channels: first we consider a discrete channel where the transmission randomly alternates between two different levels, and second we consider the semicontinuous channel where the transmission alternates between 45 different levels with certain probability amplitudes. The probability distributions of the transmittance for the discrete channel and the continuous channel are shown in Figs $3 \mathrm{~b}$ and $4 \mathrm{~b}$, respectively.

The discrete channel alternates between full transmission and $25 \%$ transmission, each realization occurring with a probability of $50 \%$. After transmission in such a channel the resulting state is a mixture of a highly and a weakly entangled state. For this state we measure the Gaussian LN to be $-1.63 \pm 0.02$. The Gaussian entanglement is thus completely lost as a result of the introduction of time-dependent loss.

The state is then fed into the distiller and we perform homodyne measurements of beam A, beam B and the tap beam simultaneously. The statistics of the quadrature measurements on the tap beam and beam $B$ as well as the joint distribution of $\hat{X}_{\mathrm{A}}+\hat{X}_{\mathrm{B}}$ and $\hat{P}_{\mathrm{A}}-\hat{P}_{\mathrm{B}}$ are shown in Fig. 2 . From the narrowing of the joint distributions to below that of the shot noise, we conclude qualitatively that Gaussian entanglement has been recovered.

The Gaussian LN has been computed for several choices of the threshold value $X_{\text {th }}$, and is plotted in Fig. 3 as a function of the associated success probabilities. Furthermore, the probability coefficients of the two states in the mixture after distillation are 
a
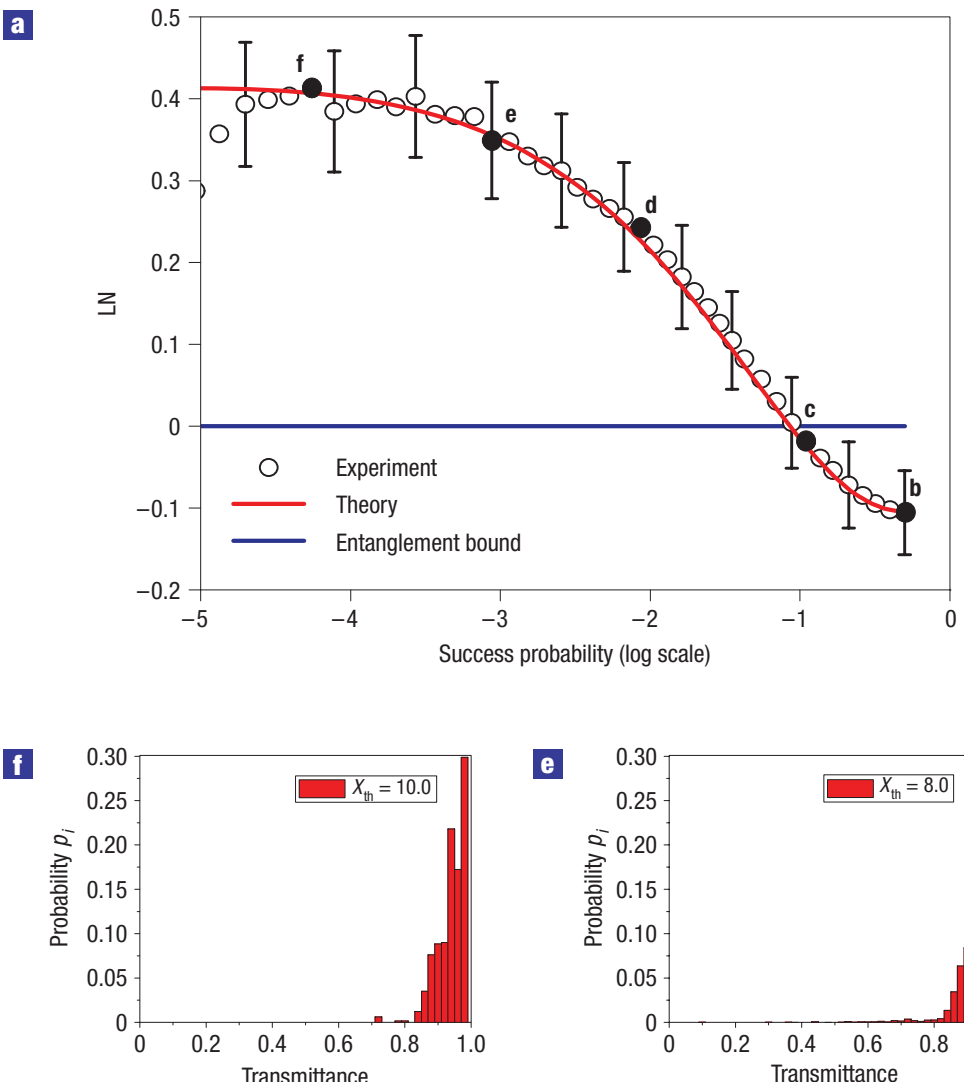

e

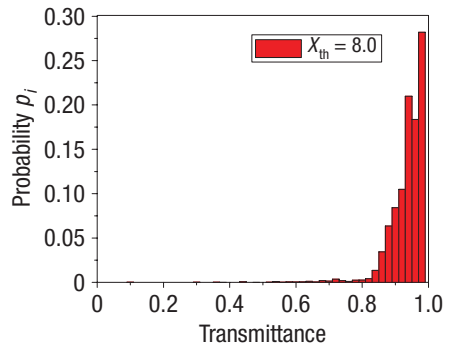

b

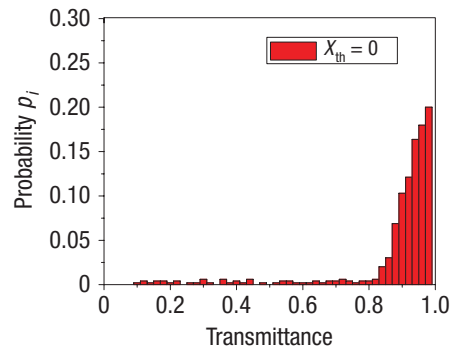

$\boldsymbol{C}$

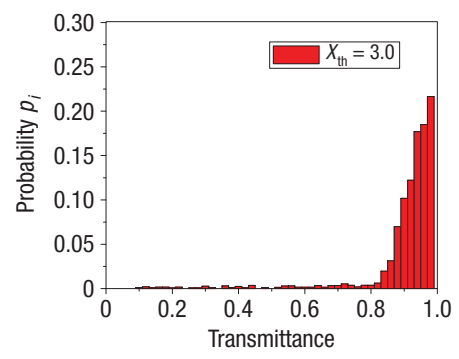

d

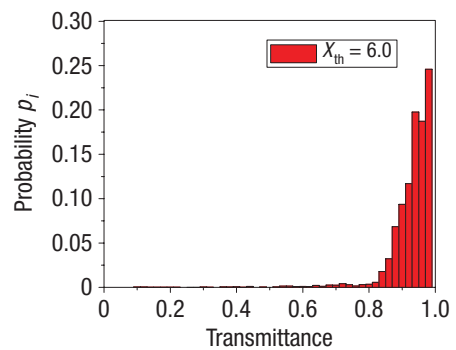

Figure 4 Experimental and theoretical results outlining the distillation of an entangled state from a semicontinuous lossy channel. a, The experimental results are marked by circles and the theoretical prediction by the red solid curve. The error bars are determined in the same way as described in the caption of Fig. 3. b-f, The evolution of the mixture is directly visualized in a series of probability distributions. We see that for $X_{\mathrm{th}}=10$ shot noise units the probabilities associated with low transmission levels are zero and the probability for full transmission has increased to $30 \%$, as opposed to $20 \%$ before distillation. It is thus clear that the highly entangled states in the mixture have larger weight after distillation.

shown for different postselection thresholds. Note that, as the threshold increases, the mixture of the two Gaussian states reduces to a single highly entangled Gaussian state, thus demonstrating the act of Gaussification. Based on the experimental parameters the theoretical predictions are computed and illustrated in the figure by the red curve, which is seen to be in good agreement with the experimental results.

The results clearly show that the amount of Gaussian entanglement is increased by the distillation operation. To estimate whether the total entanglement is increased, we compute the upper bound for the LN before distillation and verify that this bound can be surpassed by the Gaussian LN after distillation (see the Methods section). The upper bound of LN without the Gaussian approximation is computable from the LN of each Gaussian state in the mixture ${ }^{20}$ and we find $\mathrm{LN}_{\text {upper }}=0.49$, which is shown in Fig. 3 by the dashed black line. We see that for a success probability around $10^{-4}$ the Gaussian LN crosses the upper bound for entanglement, and as the state at this point is Gaussified we may conclude that the total entanglement of the state has indeed increased as a result of the distillation.

Entanglement distillation also comes with a cost. As the degree of entanglement is increasing, the number of distilled data, or equivalently the success probability, decreases. For example, when the postselection threshold is $X_{\text {th }}=9$ shot noise units, the Gaussian $\mathrm{LN}$ is $0.67 \pm 0.09$ and the success probability is $1.69 \times 10^{-5}$. The
Table 1 Measured Gaussian LN before and after distillation and the corresponding success rates.

\begin{tabular}{llll}
\hline Channels & LN (before) & LN (after) & Success rate $P_{\mathrm{S}}$ \\
\hline Perfect & $0.76 \pm 0.08$ & $0.76 \pm 0.08$ & 1 \\
Discrete & $-1.63 \pm 0.02$ & $0.67 \pm 0.09$ & $1.69 \times 10^{-5}$ \\
Semicontinuous & $-0.11 \pm 0.04$ & $0.39 \pm 0.07$ & $1.66 \times 10^{-5}$
\end{tabular}

protocol has extracted only 816 highly entangled states from a total set of $2.4 \times 10^{7}$ less entangled states.

We now turn our attention to a communication channel that takes on 45 different transmission levels as opposed to the twolevel channel. The distribution of the transmittance is illustrated in Fig. 4b. This channel simulates a free-space optical communication channel where atmospheric turbulence causes scattering and beampointing noise ${ }^{19}$. After propagation through this channel the Gaussian LN of the mixed state is found to be $-0.11 \pm 0.05$, which is substantially lower than the original value of $0.76 \pm 0.08$. The state is subsequently distilled and the change in the Gaussian LN as the threshold value increases (and the success probability decreases) is shown in Fig. 4a. We clearly see the trend that the entanglement available for Gaussian operations is increased, ultimately reaching the level of $\mathrm{LN}=0.39 \pm 0.07$. 
A summary of the measured values for $\mathrm{LN}$ in the various channels before and after distillation is presented in Table 1 . The demonstration of a distillation protocol in this letter provides a crucial step towards the construction of a quantum repeater ${ }^{27}$ for transmitting continuous-variable quantum states over long distances in channels afflicted by non-Gaussian noise. The various ingredients for a continuous-variable quantum repeater that could potentially overcome non-Gaussian noise-a quantum memory ${ }^{28}$, a teleportation protocol $^{29}$ and an entanglement distillation protocol-have now all been experimentally realized and the next step is to combine some of these technologies.

\section{METHODS}

\section{THEORY OF DISTILLATION OPERATION}

Theoretically, the distilled state reads

$$
\begin{aligned}
W_{p}\left(X_{\mathrm{A}}, P_{\mathrm{A}}, X_{\mathrm{B}}, P_{\mathrm{B}}\right)= & \int_{X_{\mathrm{th}}}^{\infty} \int_{-\infty}^{\infty} \sum_{i=1}^{N} p_{i} W_{i}^{\prime}\left(X_{\mathrm{A}}, P_{\mathrm{A}}, X_{\mathrm{B}}, P_{\mathrm{B}}\right) \\
& \times W_{0}\left(X_{T}, P_{T}\right) \mathrm{d} X_{T}^{\prime} \mathrm{d} P_{T}^{\prime},
\end{aligned}
$$

where $X_{\mathrm{B}}^{\prime}=\sqrt{T} X_{\mathrm{B}}-\sqrt{1-T} X_{T}, P_{\mathrm{B}}^{\prime}=\sqrt{T} P_{\mathrm{B}}-\sqrt{1-T} P_{T}$, $X_{T}^{\prime}=\sqrt{T} X_{T}+\sqrt{1-T} X_{\mathrm{B}}$ and $P_{T}^{\prime}=\sqrt{T} P_{T}+\sqrt{1-T} P_{\mathrm{B}}$, and the Wigner function $W_{0}\left(X_{T}, P_{T}\right)$ represents the vacuum mode entering the asymmetric beam splitter with transmittance $T$. The question we now seek to answer is whether this state is more entangled than the predistilled state. The above-mentioned measure - the Gaussian LN (equation (1)) — is valid as an entanglement monotone only if the state is Gaussian, which is not the case for all stages of our experiment. However, the Gaussian LN is a good measure of entanglement useful for Gaussian operations. An example is continuousvariable quantum teleportation, where an increase in the Gaussian LN is directly linked with an increase in the teleportation fidelity ${ }^{30}$. To prove that the total entanglement of the state has increased as a result of distillation, we compute an upper bound for the $\mathrm{LN}$ before distillation $\left(\mathrm{LN}=\log _{2}\left\|\rho^{T}\right\|_{1}\right.$, where $\rho^{T}$ is the partial transposed density matrix of the state) and demonstrate that this bound can be surpassed by the Gaussian LN when the state is Gaussified after distillation (as the measure then becomes exact).

\section{GENERATION OF ENTANGLEMENT}

The polarization-squeezed beams are produced by launching two femtosecond pulses with balanced powers onto the two orthogonal polarization modes of two different fibres. We use two 13.2-m-long polarization-maintaining fibres. The pump source is a $\mathrm{Cr}^{4+}: \mathrm{YAG}$ laser at a wavelength of $1,500 \mathrm{~nm}$, repetition rate of $163 \mathrm{MHz}$. The two orthogonal polarization modes in each fibre are squeezed and temporally overlapped with a relative phase of $\pi / 2$, thus producing a circularly polarized light beam represented by the Stokes parameter $S_{3}$. The relative phase is achieved and controlled using an interferometric birefringence compensation and a locking loop based on $0.1 \%$ of the fibre output. Since the optical excitation is along $S_{3}$, the orthogonal Stokes plane spanned by the Stokes parameters $S_{1}$ and $S_{2}$ is 'dark'. However, it contains a vacuum-squeezed state, which can be easily measured in a polarization analyser using the orthogonally polarized excitation. Polarization squeezing in the 'dark' plane is thus equivalent to quadrature vacuum squeezing. We thus set $\hat{S}\left(\theta_{\mathrm{sq}}\right) \rightarrow \hat{X}$ and $\hat{S}\left(\theta_{\mathrm{sq}}+\pi / 2\right) \rightarrow \hat{P}$, where $\hat{S}\left(\theta_{\mathrm{sq}}\right)$ and $\hat{S}\left(\theta_{\mathrm{sq}}+\pi / 2\right)$ are the 'dark' Stokes operators of the squeezing and antisqueezing directions in the dark plane. The two squeezed beams interfere on a 50/50 beam splitter to produce entanglement and we subsequently measure all second-order moments between the quadratures using two homodyne detectors at $17 \pm 0.5 \mathrm{MHz}$.

\section{MEASUREMENT OF ENTANGLEMENT}

A general polarization, Stokes, measurement apparatus consists of a half-wave plate, a polarizing beam splitter and two intensity detectors. The half-wave plate enables the measurement of different Stokes parameters lying in the 'dark' plane when the light beam is circularly $\left(S_{3}\right)$ polarized. The polarizing beam splitter outputs are measured directly by using intensity detectors with 98\%-quantum-efficiency InGaAs photodiodes and with an incorporated low-pass filter to avoid a.c. saturation due to the laser repetition oscillation.
The difference current from the two detectors is produced and subsequently mixed with an electronic local oscillator at $17 \mathrm{MHz}$, low-pass filtered $(1.9 \mathrm{MHz})$, amplified (FEMTO DHPVA-100) and finally digitized by an A/D exit converter (Gage CompuScope 1610) at $10^{7}$ samples per second with a 16-bit resolution. After these data processing steps, the noise statistics of the Stokes parameters are characterized at $17 \mathrm{MHz}$ relative to the optical field carrier frequency with a bandwidth of $1 \mathrm{MHz}$. The signal is sampled around this sideband to avoid the classical noise present in the frequency band around the carrier. The measurement of polarization entanglement is accomplished by applying identical polarization measurements on beams A and B with the half-wave plates set to the same angle (either measuring $\hat{X}$ or $\hat{P}$ in the 'dark' polarization plane). For each angle, the detected photocurrent noise of beams A and B was simultaneously sampled $2.4 \times 10^{8}$ times, and the self- and cross-correlations between the data set could easily be calculated. The covariance matrix was subsequently determined and the $\mathrm{LN}$ was calculated.

Received 15 April 2008; accepted 26 September 2008; published 2 November 2008.

\section{References}

1. Ekert, A. K. Quantum cryptography based on Bell theorem. Phys. Rev. Lett. 67, 661-663 (1991).

2. Bennettt, C. H. et al. Teleporting an unknown quantum state via dual classical and Einstein-Podolsky-Rosen channels. Phys. Rev. Lett. 70, 1895-1899 (1992).

3. Bennettt, C. H. \& Wiesner, S. J. Communication via one- and two-particle operators on Einstein-Podolsky-Rosen states. Phys. Rev. Lett. 69, 2881-2884 (1992).

4. Bennett, C. H. et al. Purification of noisy entanglement and faithful teleportation via noisy channels. Phys. Rev. Lett. 76, 722-725 (1996).

5. Kwiat, P. G. et al. Experimental entanglement distillation and hidden non-locality. Nature $\mathbf{4 0 9}$, 1014-1017 (2001)

6. Pan, J.-W., Gasparoni, S., Ursin, R., Weihs, G \& Zeilinger, A. Experimental entanglement purification of arbitrary unknown states. Nature 423, 417-422 (2003).

7. Eisert, J., Scheel, S. \& Plenio, M. B. Distilling Gaussian states with Gaussian operations is impossible. Phys. Rev. Lett. 89, 137903 (2002).

8. Fiurášek, J. Gaussian transformations and distillation of entangled Gaussian states. Phys. Rev. Lett. 89, 137904 (2002).

9. Giedke, G. \& Cirac, J. I. Characterization of Gaussian operations and distillation of Gaussian states. Phys. Rev. A 66, 032316 (2002).

10. Opatrný, T., Kurizki, G. \& Welsch, D. G. Improvement on teleportation of continuous variables by photon subtraction via conditional measurement. Phys. Rev. A 61, 032302 (2000).

11. Duan, L.-M., Giedke, G., Cirac, J. I. \& Zoller, P. Entanglement purification of Gaussian continuous variable quantum states. Phys. Rev. Lett. 84, 4002-4005 (2000).

12. Browne, D. E. \& Eisert, et al. Driving non-Gaussian to Gaussian states with linear optics. Phys. Rev. A 67, 062320 (2003).

13. Fiurášek, J., Mišta, L. Jr. \& Filip, R. Entanglement concentration of continuous-variable quantum states. Phys. Rev. A 67, 022304 (2003).

14. Ourjoumtsev, A., Dantan, A., Tualle-Brouri, R. \& Grangier, P. Increasing entanglement between Gaussian states by coherent photon subtraction. Phys. Rev. Lett. 98, 030502 (2007).

15. Fiorentino, M. et al. Soliton squeezing in a Mach-Zehnder fiber interferometer. Phys. Rev. A 64, 031801 (2001).

16. Dong, R.-F. et al. An efficient source of continuous variable polarisation entanglement. New J. Phys. 9, 410 (2007).

17. Dong, R.-F. et al. Experimental evidence for Raman-induced limits to efficient squeezing in optical fibers. Opt. Lett. 33, 116-118 (2008).

18. Ursin, R. et al. Entanglement-based quantum communication over $144 \mathrm{~km}$. Nature Phys. 3, $481-486$ (2007).

19. Majumdar, A. K. \& Ricklin, J. C. Free-Space Laser Communications (Springer, 2008).

20. Vidal, G. \& Werner, R. F. Computable measure of entanglement. Phys. Rev. A 65, 032314 (2002).

21. Laurat, J. et al. Conditional preparation of a quantum state in the continuous variable regime: Generation of a sub-poissonian state from twin beams. Phys. Rev. Lett. 91, 213601 (2003).

22. Heersink, J. et al. Experimental distillation of squeezing from non-Gaussian quantum states. Phys. Rev. Lett. 96, 253601 (2006).

23. Franzen, A., Hage, B., DiGuglielmo, J., Fiurášek, J. \& Schnabel, R. Experimental demonstration of continuous variable purification of squeezed states. Phys. Rev. Lett. 97, 150505 (2006).

24. Suzuki, S., Takeoka, M., Sasaki, M., Andersen, U. L. \& Kannari, F. Practical purification scheme for decohered coherent-state superpositions via partial homodyne detection. Phys. Rev. A 73, 042304 (2006).

25. Andrew, M. L. et al. Quantum state engineering with continuous-variable post-selection. Phys. Rev. A 73, 041801 (R) (2006)

26. Fiurášek, J., Marek, P., Filip, R. \& Schnabel, R. Experimentally feasible purification of continuous-variable entanglement. Phys. Rev. A 75, 050302 (2007).

27. Briegel, H.-J., Dür, W., Cirac, J. I. \& Zoller, P. Quantum repeaters: The role of imperfect local operations in quantum communication. Phys. Rev. Lett. 81, 5932-5935 (1998).

28. Julsgaard, B. et al. Experimental demonstration of quantum memory for light. Nature 432, 482-486 (2004).

29. Furusawa, A. et al. Unconditional quantum teleportation. Science 282, 706-709 (1998).

30. Adesso, G. \& Illuminati, F. Phys. Rev. Lett. 95, 150503 (2005).

\section{Acknowledgements}

This work was supported by the EU project COMPAS (No. 212008), the Deutsche Forschungsgesellschaft and the Danish Agency for Science Technology and Innovation (No. 274-070509). M.L. and R.F. acknowledge support from the Alexander von Humboldt Foundation and R.F. acknowledges Measurement and Information in Optics (MSM6198959213), LC 06007 of the Czech Ministry of Education and 202/07/J040 of GACR.

\section{Author information}

Reprints and permissions information is available online at http://npg.nature.com/reprintsandpermissions. Correspondence and requests for materials should be addressed to U.L.A. 